\title{
A Plastic Load Criterion for Inelastic Design by Analysis
}

\author{
Donald Mackenzie and Hongjun Li \\ Department of Mechanical Engineering \\ University of Strathclyde \\ Glasgow \\ United Kingdom
}

Tel. (44) 1415482046

d.mackenzie@strath.ac.uk

hongjun.li.100@strath.ac.uk

\section{ABSTRACT}

Background. The allowable plastic load in pressure vessel Design by Analysis is determined by applying a graphical construction to a characteristic load-deformation plot of the collapse behavior of the vessel. This paper presents an alternative approach to the problem.

Method of Approach. The plastic response is characterized by considering the curvature of a plot of plastic work dissipated in the vessel against the applied load. It is proposed that salient points of curvature correspond to critical stages in the evolution of the gross plastic deformation mechanism. In the proposed Plastic Work Curvature criterion of plastic collapse, the plastic load is defined as the load corresponding to zero or minimal plastic work curvature after yielding and the formation of plastic mechanisms have occurred.

Results. Application of the proposed criterion is illustrated by considering the elastic-plastic response of a simple cantilever beam in bending and a complex 3-D Finite Element Analysis of a nozzle intersection.

Conclusions. The results show that the proposed approach gives higher values of plastic load than alternative criteria when the material exhibits strain hardening. It is proposed that this is because the PWC criterion more fully represents the constraining effect of material strain hardening on the spread of plastic deformation.

Keywords. Design by Analysis, criterion of plastic collapse, gross plastic deformation, strain hardening. 


\section{INTRODUCTION}

The ASME B\&PV Code Section VIII Division 2 Appendix 4 [1] provides guidelines for Design by Analysis (DBA) based on elastic and inelastic stress analysis. When elastic analysis is used, gross plastic deformation is prevented by limiting the primary stress in the vessel. When inelastic analysis is used, gross plastic deformation is prevented by restricting the allowable load with respect to either the limit load or the plastic load of the vessel. The limit load is the maximum load satisfying equilibrium between external and internal forces when an elasticperfectly plastic material model and small deformation theory are assumed. The plastic load is based on a more complex analysis, which may include large deformation effects and/or material strain hardening. When large deformations are significant, the vessel may exhibit geometrical strengthening or weakening. When strain hardening is included, plastically deformed material can support stresses greater than yield, enhancing the strength of the vessel. In ASME DBA, the plastic load is defined by applying the Twice Elastic Slope (TES) criterion of plastic collapse to a characteristic load-deformation curve for the vessel. In the TES criterion, the structural response is characterized by plotting a load parameter against a deformation parameter. A straight collapse limit line is then drawn from the origin of the characteristic curve with slope conventionally referred to as twice the slope of the initial elastic response relative to the load axis. This corresponds to half the stiffness of the initial response, as shown in Fig. 1. The plastic load, $\mathrm{P}_{\mathrm{P}}$, is defined as the load corresponding to the intersection of the collapse limit line and the load-deformation curve.

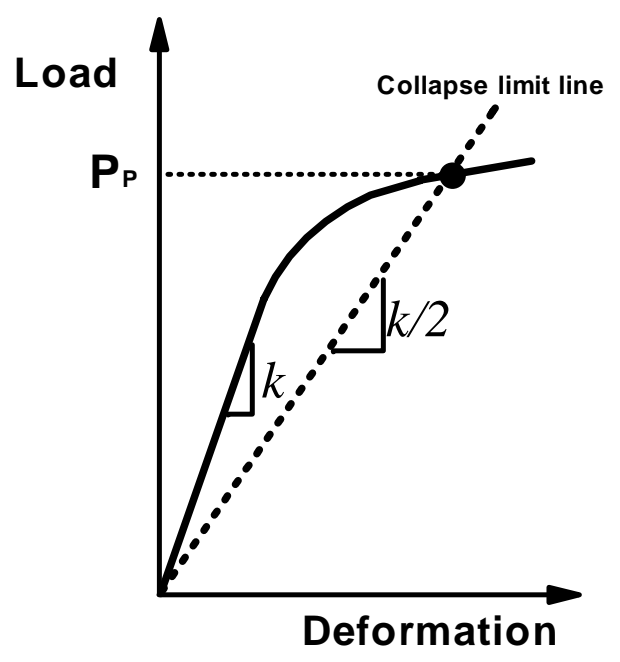

Figure 1. Twice Elastic Slope, TES, criterion. 
The TES criterion is a heuristic criterion originating in experimental DBA [2]. It is to some extent arbitrary and does not relate the specified plastic load to any specific event in the evolution of the plastic deformation mechanism.

In practice, the load parameter is usually the most significant load acting on the vessel but this can be difficult to define for vessels subject to several combined loads. The deformation parameter may be displacement, rotation or strain at a point on the vessel. This local parameter is required to adequately represent the development of global plastic failure mechanisms and the location and nature of deformation parameter chosen can significantly affect the calculated plastic pressure. Gerdeen [2] proposed that the load and deformation should be chosen such that their product has units of work, Nm. He does not recommend the use of strain as a deformation parameter as he found this usually resulted in over-conservative design.

The TES criterion has several practical difficulties. As well as problems in selecting the appropriate load parameter for combined loading, in some cases there is no intersection between the load-deformation curve and collapse limit line [2,3]. Moffat et al [4] identified another problem when investigating plastic collapse of branch connections. Their experimental and Finite Element Analysis (FEA) studies showed the TES criterion did not give a unique value for plastic load, as it is influenced by the elastic response of the structure remote from the region where the plastic failure mechanism actually occurs. This is a significant disadvantage when DBA is based on inelastic FEA, as analysts routinely model regions of a vessel (with appropriate boundary conditions) rather than the complete structure.

Several other plastic criteria similar to the TES criterion have been proposed in the literature but all exhibit similar practical limitations. Moffat et al considered one of these, the Tangent Intersection (TI) criterion [5], in his study of branch intersections. In the TI criterion, two straight lines are drawn on the characteristic loaddeformation curve, one tangent to the initial elastic response and one tangent to the plastic deformation region of the curve, as shown in Fig. 2. The load corresponding to the intersection of the two straight lines is defined as the plastic load. Moffat et al found that the TI criterion gives a unique value for the plastic load of branch intersections, independent of the elastic response of the structure. However, the TI criterion has the practical disadvantage that it is often unclear as to where the tangent to the plastic portion of the curve should be drawn [3]. 


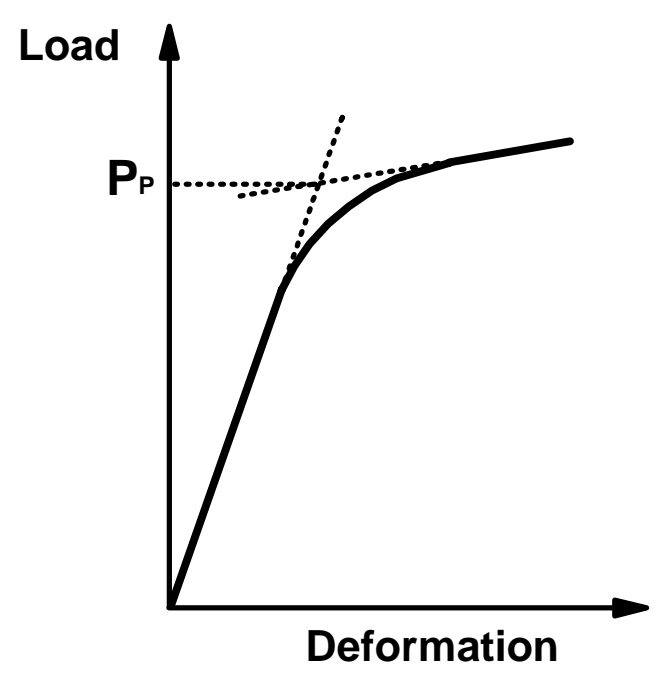

Figure 2. Tangent Intersection, TI, criterion.

Gerdeen attempted to set the definition of plastic load on a theoretical basis by proposing that the plastic load could be determined by considering the relationship between the elastic strain energy stored and plastic work dissipated in the structure. He proposed that the plastic load is reached when the plastic work is some factor $\alpha$ of the elastic strain energy stored, however he did not define a general value for $\alpha$ but referred to earlier criteria for suitable values for particular applications. Muscat et al [6] adopted a different approach and proposed a plastic collapse criterion based on a characteristic plot of a load parameter, $\lambda$, representing all applied loads, against plastic work dissipation in the vessel. The total plastic work dissipated in the vessel is a measure of the global response and is suitable for characterizing gross plastic deformation. Muscat's Plastic Work (PW) criterion defines the plastic load as that causing excessive plastic dissipation in the vessel. The plastic load is defined as the intersection between a straight-line tangent from the plastic work curve and the load axis, as illustrated in Fig. 3. 


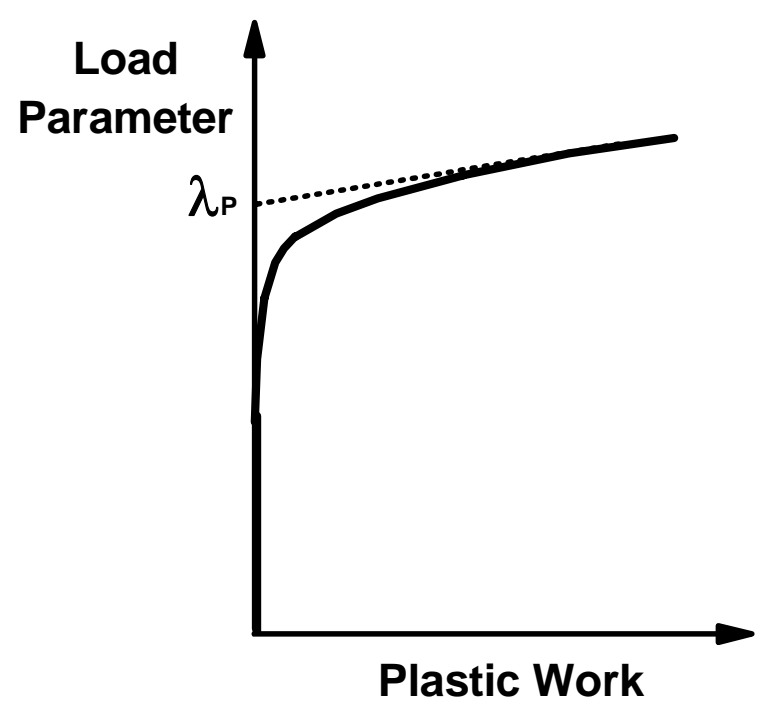

Figure 3. Plastic Work, PW, criterion.

The PW criterion has practical advantages over alternative criteria. The characteristic structural response curve is defined for all applied loads and the plastic work parameter represents the global plastic deformation of the structure. Unlike the TES criterion, the PW criterion plastic loads are determined purely by the inelastic response of the structure and are not influenced by the initial elastic response, giving a unique plastic load for each structure considered and, in finite element analysis, allows low stress regions of the structure to be omitted from the model and represented by suitable boundary conditions without affecting the calculated limit or plastic load. However, the PW criterion does not specify an appropriate point from which to draw the tangent to the plastic response curve.

Here, a new plastic criterion based on plastic work concepts is proposed. The new criterion has several features in common with the PW criterion. The response is characterized by considering the relationship between load and plastic work and a load parameter is used for combined loads. The plastic load is defined by relating the curvature of the load-plastic work curve to specific stages in the evolution of the gross plastic deformation mechanism. The criterion is therefore referred to as the Plastic Work Curvature or PWC criterion. 


\section{THE PWC CRITERION}

The PWC criterion is introduced by considering the response of a rectangular cantilever beam of bilinear hardening material under bending. The beam dimensions are width $b=10 \mathrm{~mm}$, depth $d=10 \mathrm{~mm}$, length $L n=50 \mathrm{~mm}$. The bilinear strain hardening material properties are Young's modulus E=200 GPa, Poisson's Ratio $v=0.3$, yield stress $\sigma_{\mathrm{y}}=300 \mathrm{MPa}$, with plastic modulus $E_{p l}=0 \mathrm{GPa}$ for limit analysis and $E_{p l}=4 \mathrm{GPa}$ for strain hardening analysis. The beam is fully fixed at one end and loaded by a pure bending moment $M$ at the other, as shown in Fig. 4. FEA was performed using the ANSYS program [7]. ANSYS does not give total plastic work as a standard result, so a macro program was written to calculate it for 3D solid isoparametric elements, SOLID95 (allowing both 2-D and 3-D problems to be considered using the same macro). Convergence studies were performed for elastic analysis and an appropriately element mesh density selected. Inelastic analysis was based on the von Mises yield criterion and associated flow rule.

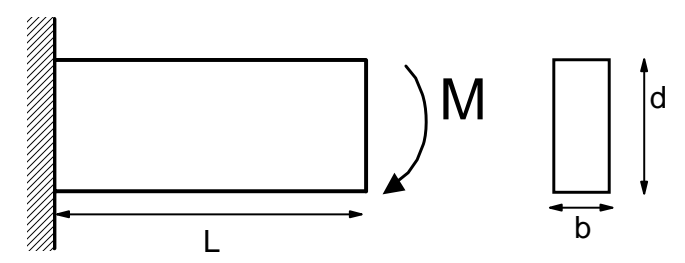

Figure 4. Cantilever beam example structure.

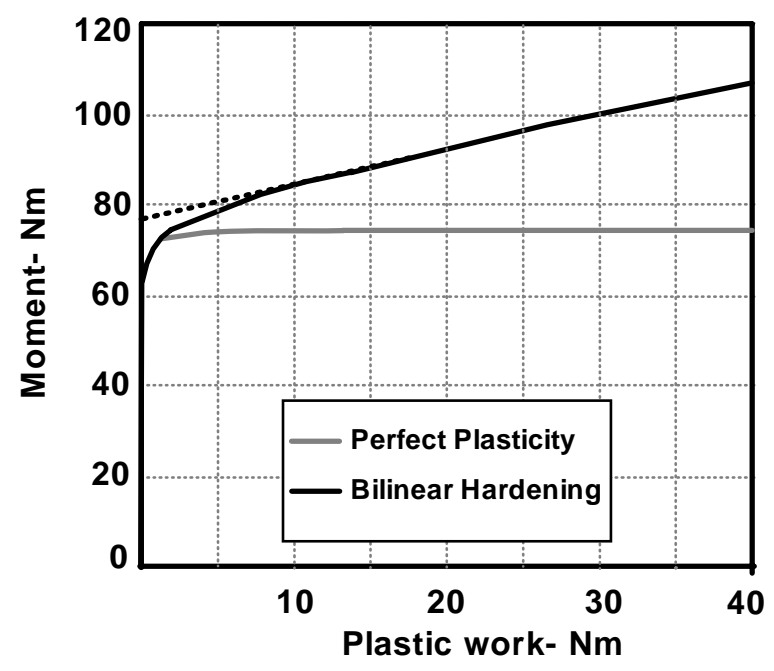

Fig 5. Beam Moment-Plastic Work curve. 
The PWC criterion defines the plastic load by considering the curvature of the load-plastic work curve obtained for the beam and shown in Fig. 5. The convention in DBA is to plot the deformation parameter (or plastic work) on the x-axis and the load on the y-axis. In defining the PWC criterion, it is useful to invert this and plot plastic work against load, as illustrated in Fig. 6. The slope of the plastic work-load curve characterizes the rate of change of plastic deformation as load increases. The rate of change of slope, and the associated curvature, characterizes how rapidly the degree of plastic deformation is changing with increasing load. In the elastic region, the curvature is zero. After yielding, plastic stress redistribution begins and the curvature increases to a maximum as the plastic deformation mechanism develops. The maximum stress redistribution occurs at the load corresponding to the maximum curvature and thereafter decreases, indicating decreasing stress redistribution. As the curvature decreases to a minimum or zero, little or no further plastic stress redistribution occurs unless a second plastic deformation mechanism is initiated. At this stage the structure is exhibiting constant, gross plastic deformation and, in the PWC criterion, the corresponding load is designated the plastic load for DBA.

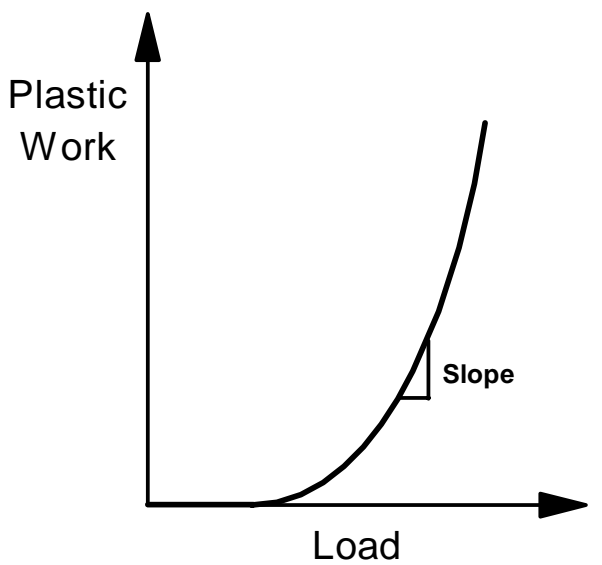

Figure 6. Characteristic plot of load against plastic work.

In the PWC criterion, load is plotted against plastic work in the conventional manner (the curvature distribution is the same as for a work-load plot). The curvature of the load-plastic work can be obtained in several ways. Here, for convenience, the commercial modeling program Pro/Engineer [8] was used to evaluate the curvature by creating a cubic spline fit through load-plastic work data points and using the ProE Curvature function. 
The limit moment of the beam, the last converged equilibrium solution in limit analysis, was $\mathrm{M}_{\mathrm{L}}=75 \mathrm{Nm}$. At the limit load, $100 \%$ of the cross section experiences plastic deformation and the moment-plastic work curve is horizontal. Plastic moments were calculated by applying the TES and TI criteria to conventional plots of tip moment versus tip rotation for the beam. The TES and TI criteria give the same value for plastic moment, $\mathrm{M}_{\mathrm{TES}}=\mathrm{M}_{\mathrm{TI}}=74 \mathrm{Nm}$. Muscat's PW criterion was applied to the plot of moment against plastic work shown in Fig. 5 and gave a slightly higher plastic load, $\mathrm{M}_{\mathrm{PW}}=78 \mathrm{Nm}$. The values of limit load and plastic load are similar, indicating that the plastic criteria do not represent the effect of the hardening material model on the plastic load. Compared to a perfectly plastic material, bilinear hardening impedes the spread of plastic deformation and a higher load would be required to cause gross plastic deformation. At the calculated plastic loads, approximately $50 \%$ of the cross section of the hardening beam has deformed plastically, compared to $100 \%$ of the perfectly plastic beam.

Moment-plastic work plots for the limit and bilinear hardening analyses of the beam are shown in Fig. 7. The curvature of the spline fit to the FE load-work data is superimposed on the curve, allowing visualization of the development of the gross plastic deformation mechanism.
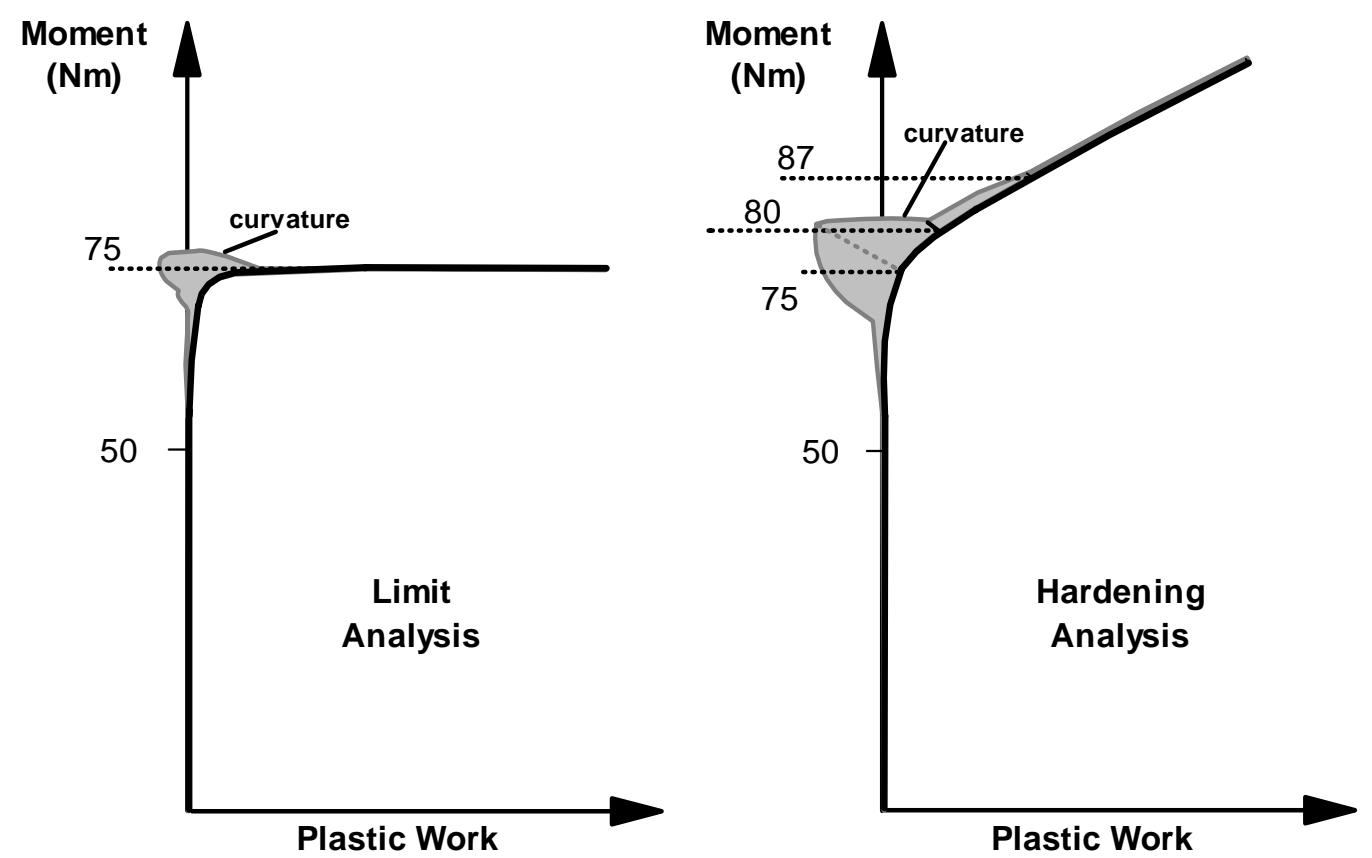

Figure 7. Moment-plastic work plot for perfectly plastic beam with curvature superimposed. 
In the perfectly plastic beam, the curvature increases from zero at yield moment $\mathrm{M}_{\mathrm{Y}}=50 \mathrm{Nm}$ to a maximum value before decreasing to zero at the PWC plastic load, $\mathrm{M}_{\mathrm{PWC}}=75 \mathrm{Nm}$, the limit load of the beam. In the bilinear hardening model, the curvature initially increases slowly from zero at first yield before increasing rapidly to a maximum value at $75 \mathrm{Nm}$. Thereafter, the curvature decreases rapidly to a discontinuity in the curvature at $\mathrm{M}=80 \mathrm{Nm}$, followed by a more gradual decrease to $\mathrm{M}_{\mathrm{PWC}}=87 \mathrm{Nm}$. At this load, $90 \%$ of the beam cross section has experienced plastic deformation. (The bilinear hardening beam approaches the fully plastic state asymptotically, hence the curvature never actually reaches zero).

The simple beam example demonstrates that the curvature of a characteristic load-plastic work curve may be used to determine the plastic load for DBA. Salient points on the curve can be related to significant events in the formation of the failure mechanism. The onset of curvature indicates initial yield, the maximum value of curvature indicates a change from elastic to plastic dominated response and the return to low curvature indicates gross plastic deformation. 


\section{BRANCH INTERSECTION EXAMPLE}

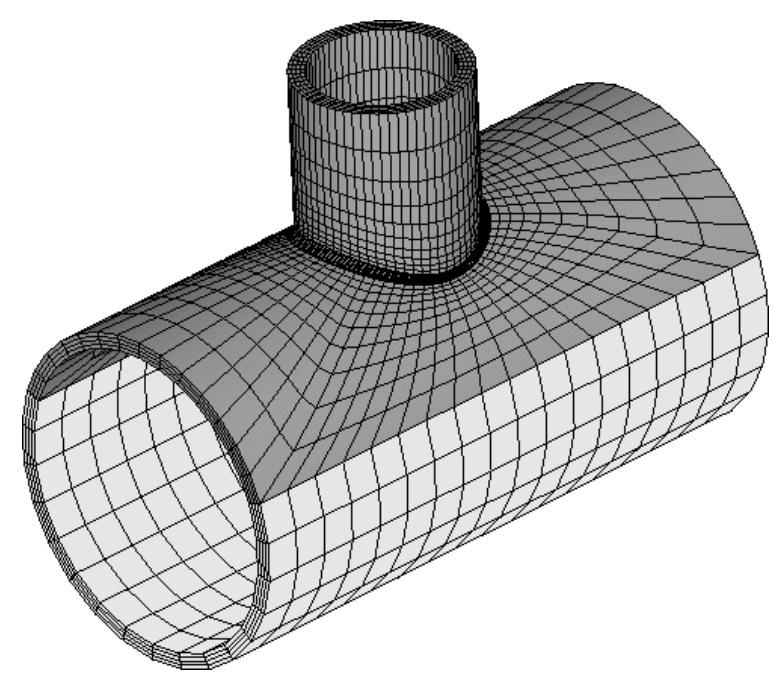

Figure 8. Finite element model of piping branch junction.

The plastic work curvature criterion was applied to a equal thickness branch intersection configuration previously investigated by Moffat et al [4] and Muscat et al [6]. The vessel geometry is shown in Fig. 8: The main run mean diameter is $D_{m}=70.67 \mathrm{~mm}$, the ratio of branch to run diameter $D_{b} / D_{m}=0.5$ and run diameter to thickness ratio $D_{b} / T=20$, with a $2.0 \mathrm{~mm}$ fillet radius between the intersecting cylinders. The main run is fully clamped at both ends. Four load cases are considered: internal pressure, in-plane bending of the branch, out-ofplane bending of the branch and combined loading, with all three loads acting. The vessel was modeled in ANSYS8.0 using 20-node brick elements SOLID95 around the junction and 8-node brick elements SOLID45 in regions remote from the junction, as shown in Fig. 8. Small and large deformation analysis was performed assuming an elastic perfectly plastic material and large deformation analysis was performed assuming a multilinear kinematic hardening material. The material yield stress $\sigma_{\mathrm{y}}=296 \mathrm{MPa}$ and Tensile Strength $\sigma_{\mathrm{TS}}=560 \mathrm{MPa}$. The kinematic material model is shown in Fig. 9. 


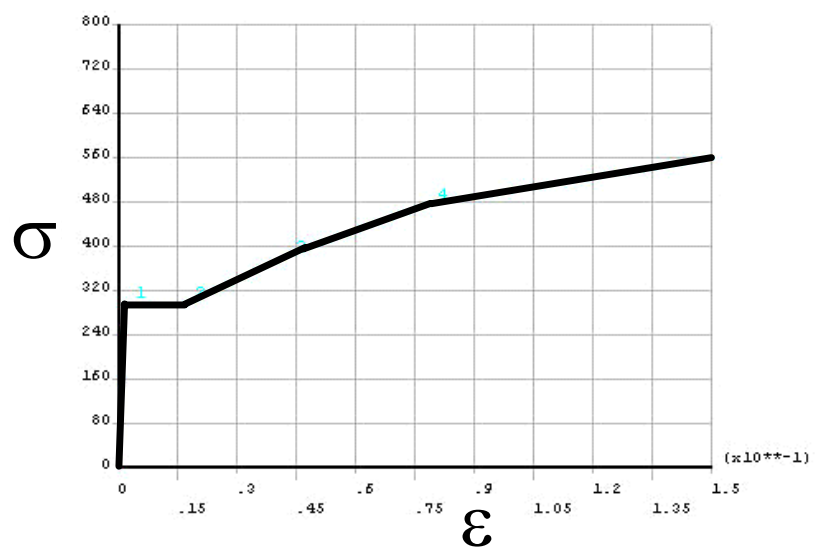

Figure 9. Multilinear hardening material model. 


\subsection{PRESSURE LOADING}

Under pressure loading, first yield occurs at the crotch corner of the intersection and spreads outwards and through the intersection. The limit analysis model, assuming perfect plasticity and small deformation theory, continued to converge for unrealistically high deformations. In such cases, a criterion of plastic collapse may be applied to define the limit load. Gerdeen [2] suggests that the TES deformation parameter corresponding to internal pressure should be change in volume of the vessel. However, change of contained volume is not a standard result available in Finite Element Analysis and the normal displacement of the nozzle end was used as the deformation parameter instead. Applying the TES to a pressure-displacement curve gave a limit pressure of $\mathrm{P}_{\mathrm{LTES}}=13 \mathrm{MPa}$. Applying the TI criterion gave a limit pressure of $\mathrm{P}_{\mathrm{LTI}}=13.5 \mathrm{MPa}$. The limit analysis momentplastic work curve and curvature, obtained from the ProE spline fit, is shown in Fig. 10. The curvature decreases rapidly from its maximum value to a discontinuity at $\mathrm{P}=13.2 \mathrm{MPa}$ and then falls to almost zero at the PWC limit pressure $\mathrm{P}_{\mathrm{LPWC}}=13.7 \mathrm{MPa}$.

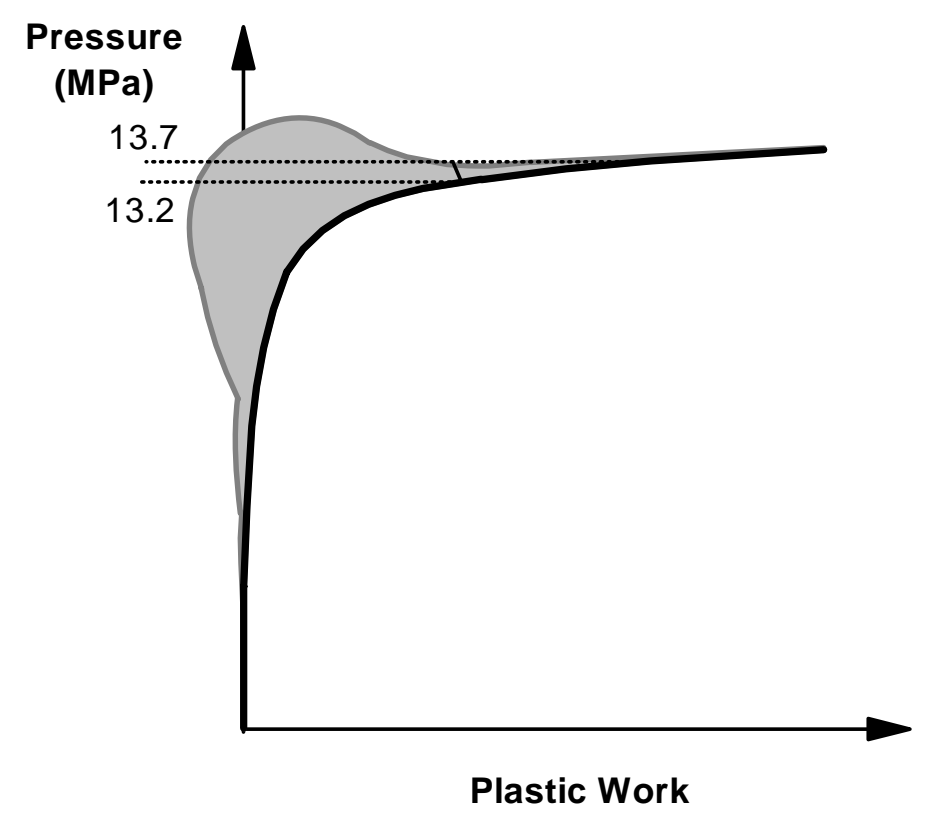

Figure 10. Limit analysis pressure-plastic work curve with curvature superimposed. 
Plastic pressures were obtained for the large deformation strain hardening analysis by applying the TES and TI criteria to a pressure-displacement curve and applying the PW criterion to the pressure-plastic work curve shown in Fig. 11. The calculated plastic pressures were $\mathrm{P}_{\mathrm{TES}}=15.5 \mathrm{MPa}, \mathrm{P}_{\mathrm{TI}}=16 \mathrm{MPa}$ and $\mathrm{P}_{\mathrm{PW}}=15.5 \mathrm{MPa}$. Unlike the beam example, the plastic criteria give plastic loads greater than the limit load. This indicates that the effect of strain hardening is captured to some degree by the criteria for this configuration. Applying the PWC criterion to Fig. 11, the plastic pressure was $\mathrm{P}_{\mathrm{PWC}}=17.1 \mathrm{MPa}$. This is higher than that given by the other criteria, indicating greater influence of strain hardening.

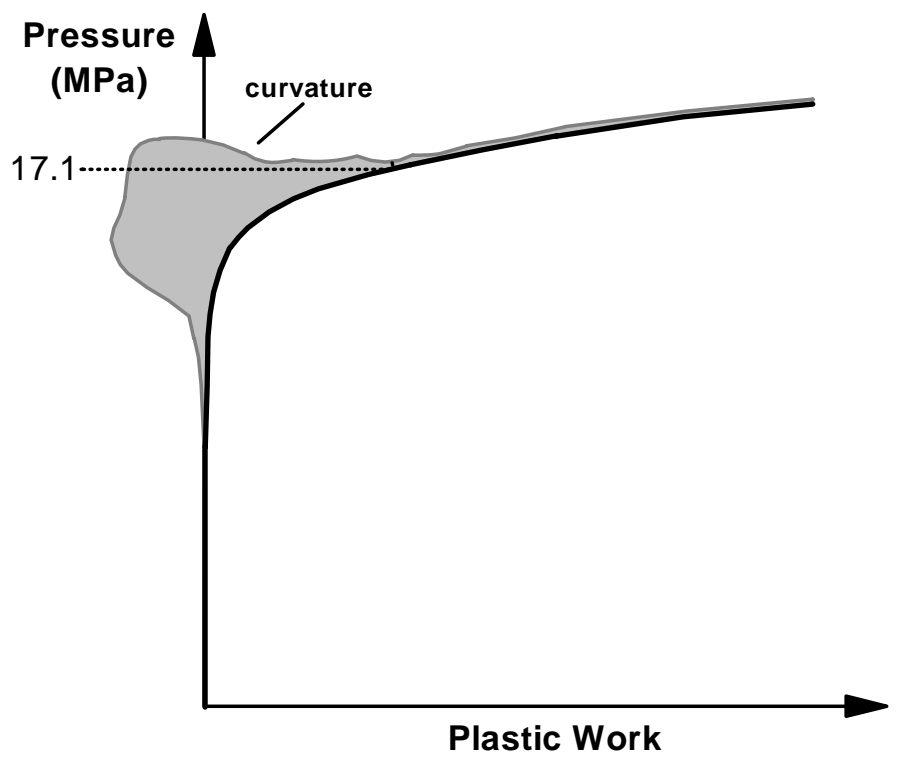

Figure 11. Plastic analysis pressure-plastic work plot with curvature superimposed. 


\subsection{IN-PLANE MOMENT}

Under in-plane moment loading, first yield occurs at the outside surfaces of the crotch corner of the junction. As the moment increases, the yield zone spreads both along the fillet to the flank of junction and towards the inside surface.

The nozzle intersection limit analysis converges for unrealistically high deformations and the TES, TI and PW criteria were applied to evaluate the limit loads of $\mathrm{M}_{\mathrm{LTES}}=900 \mathrm{Nm}, \mathrm{M}_{\mathrm{LTI}}=870 \mathrm{Nm}$ and $\mathrm{M}_{\mathrm{LPW}}=900 \mathrm{Nm}$ respectively. Applying the new PWC criterion to a moment-plastic work curvature plot gave a limit moment of $\mathrm{M}_{\mathrm{LPWC}}=940 \mathrm{Nm}$.

The plastic loads obtained by applying the plastic criteria to the large deformation strain hardening analysis were $\mathrm{M}_{\mathrm{TES}}=860 \mathrm{Nm}, \mathrm{M}_{\mathrm{TI}}=900 \mathrm{Nm}$ and $\mathrm{M}_{\mathrm{PW}}=920 \mathrm{Nm}$. The pressure-plastic work curvature plot shown in Fig. 12 shows a discontinuity in the curvature at $\mathrm{P}=970 \mathrm{MPa}$, followed by a reduction to almost zero curvature at the plastic moment, $\mathrm{M}_{\mathrm{PWC}}=1030 \mathrm{MPa}$. In this case, the curvature plot indicates that a limited amount of stress redistribution may occur above this load.

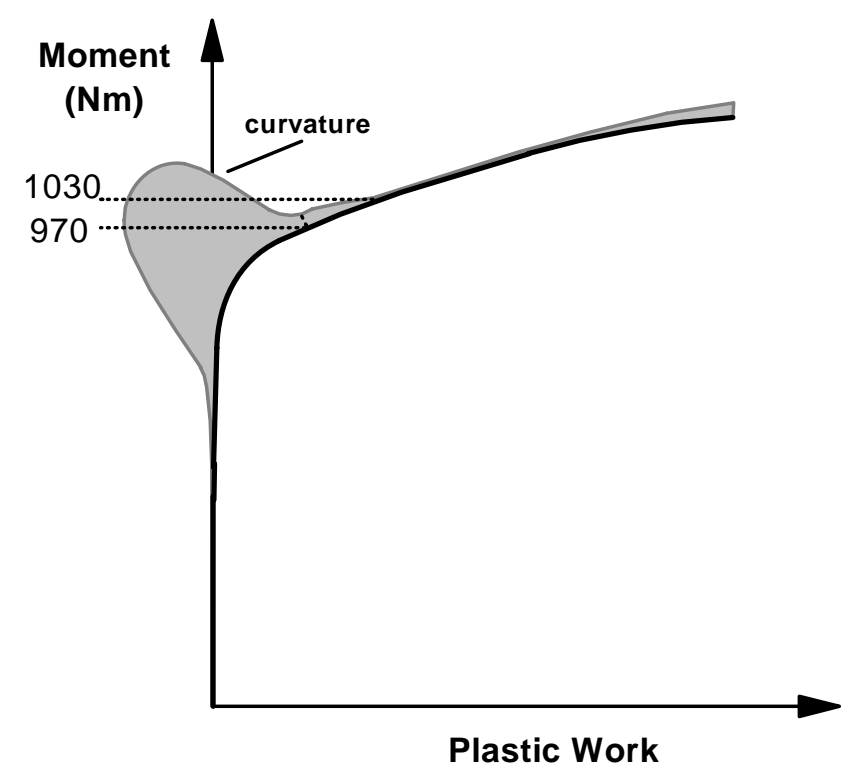

Figure 12. Plastic analysis in-plane bending- plastic curve with curvature superimposed. 


\subsection{OUT-OF-PLANE MOMENT}

Branch intersections under out-of-plane bending are known to exhibit geometric weakening [4]. Initial yield occurs at the outside surface of the flank of the junction. As the moment increases, the yield zone spreads along the fillet to the crotch corner and towards the inside surface. The maximum plastic strain remained in the first yield area during the increase of moment.

The nozzle intersection limit analysis converges for unrealistically high deformations and the plastic collapse criteria were applied to obtain the limit moments of $M_{L T E S}=610 \mathrm{Nm}, M_{L T I}=650 \mathrm{Nm}$ and $\mathrm{M}_{\mathrm{LPW}}=660 \mathrm{Nm}$. Applying the PWC criterion to a moment-plastic work curvature plot gave a limit moment of $\mathrm{M}_{\mathrm{LPWC}}=675 \mathrm{Nm}$.

The plastic loads obtained by applying the plastic criteria to the large deformation strain hardening analysis were $\mathrm{M}_{\mathrm{TES}}=720 \mathrm{Nm}, \mathrm{M}_{\mathrm{TI}}=660 \mathrm{Nm}$ and $\mathrm{M}_{\mathrm{PW}}=740 \mathrm{Nm}$. The pressure-plastic work curvature plot of Fig. 13 shows a slight discontinuity in the curvature at $\mathrm{P}=780 \mathrm{MPa}$, followed by a reduction to almost zero curvature at the plastic moment, $\mathrm{M}_{\mathrm{PwC}}=820 \mathrm{MPa}$.

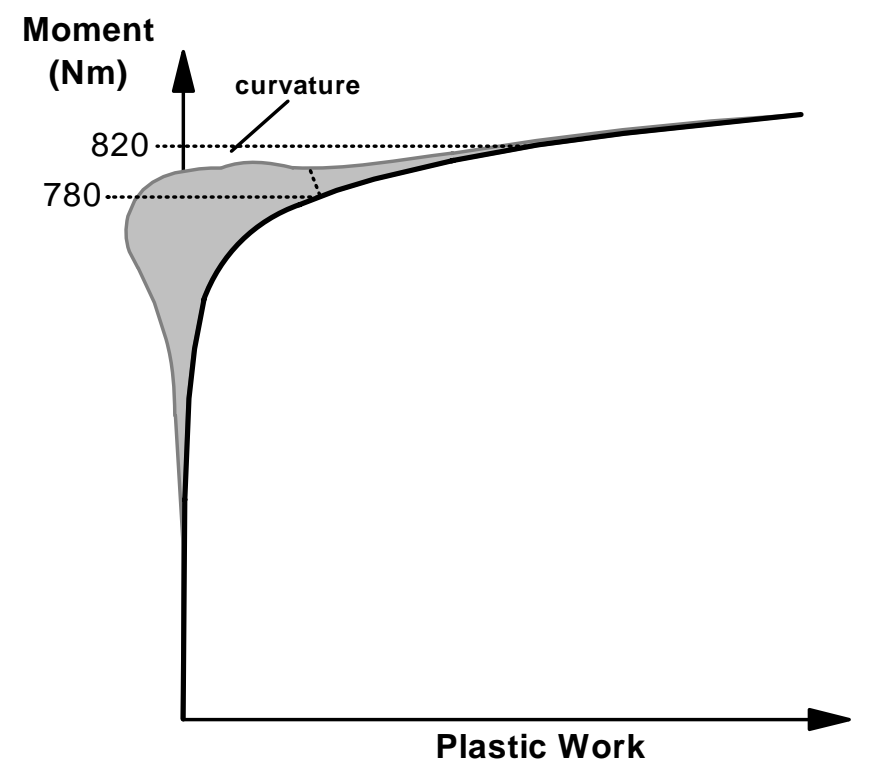

Figure 13. Plastic analysis out-of-plane bending -plastic work plot with curvature superimposed. 


\subsection{COMBINED LOADING}

Use of the PWC criterion in combined loading is illustrated by considering the branch intersection subject to combined pressure and bending loads. The proposed design loading is $P=6 \mathrm{MPa}, \mathrm{M}_{\mathrm{i}}=500 \mathrm{Nm}$ and $\mathrm{M}_{0}=400 \mathrm{Nm}$, applied by proportional loading. The design load is characterized by the proportional load parameter $\lambda\left(P, M_{i}, M_{o}\right)$, such that:

$$
\lambda(6,500,400)=1
$$

Limit analysis of the intersection converged for unrealistically high deformations and the limit load was defined by applying the PW and PWC criteria to obtain $\lambda_{\mathrm{LPW}}=1.26$ and $\lambda_{\mathrm{LPWC}}=1.28$. The DBA allowable load based on limit analysis is $2 / 3$ of the limit load. The allowable limit load parameter is therefore

$$
\lambda_{\mathrm{AL}}=\frac{2}{3} \times 1.28=0.86
$$

The specified design loads therefore exceed the allowable loads based on limit analysis. The maximum allowable loads according to limit analysis are

$$
\left(P, M_{i,} M_{o}\right)=0.86(6,500,400)
$$




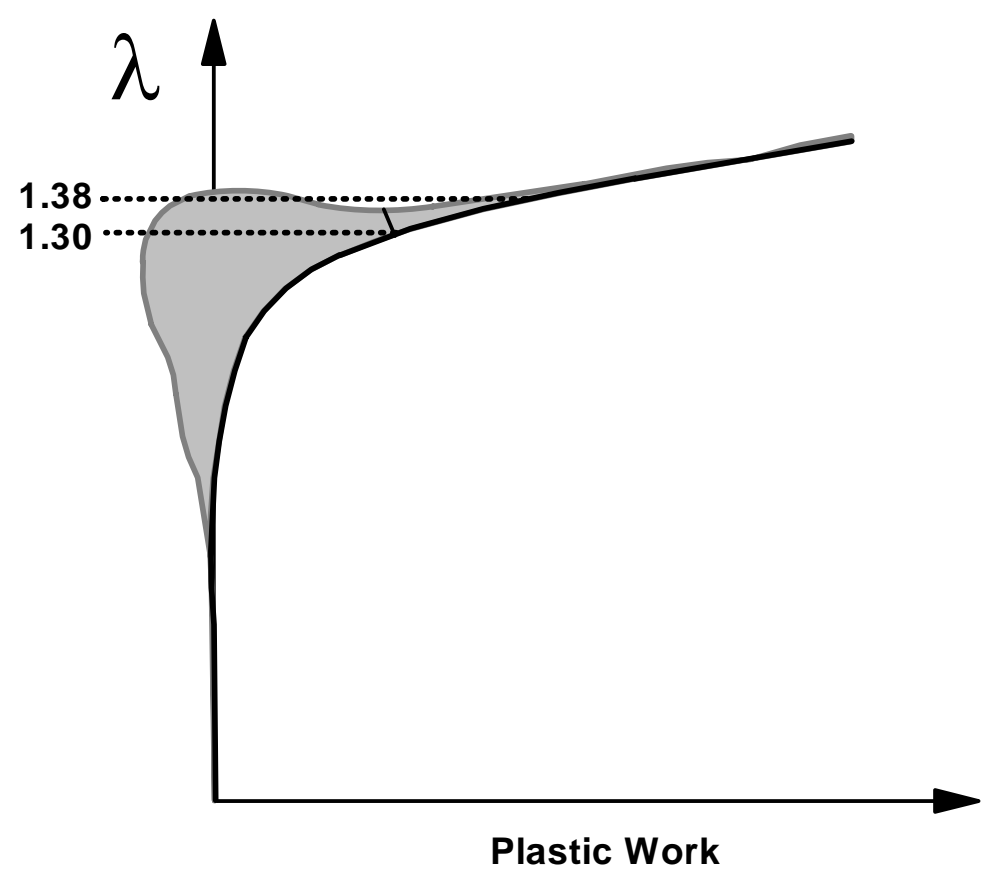

Figure 14. Plastic analysis combined loading load parameter-plastic work curve with curvature superimposed.

The pressure-plastic work curvature plot for the combined loading plastic analysis is shown in Fig. 14. Applying the PW criterion to the load-plastic work curve gives a plastic load parameter $\lambda_{\mathrm{PW}}=1.25$. Considering the curvature, there is a slight discontinuity at $\lambda=1.30$, followed by a reduction to almost zero curvature at the plastic load factor, $\lambda_{\mathrm{PWC}}=1.38$. The DBA allowable load based on plastic analysis is $2 / 3$ of the plastic load. The allowable plastic load parameter is therefore

$$
\lambda_{\mathrm{AP}}=\frac{2}{3} \times 1.38=0.92
$$

The specified design loads therefore exceed the allowable loads based on plastic analysis. The maximum allowable loads according to plastic analysis are

$$
\left(P, M_{i,} M_{o}\right)=0.92(6,500,400)
$$




\section{CONCLUSION}

Table 1. Limit and plastic loads for the nozzle intersection under pressure, in-plane moment and out-ofplane moment.

\begin{tabular}{|c|c|c|c|c|c|}
\hline Load & $\begin{array}{c}\text { Limit } \\
\text { (PWC) }\end{array}$ & TES & TI & PW & PWC \\
\hline $\begin{array}{c}\text { Pressure } \\
\text { MPa }\end{array}$ & 13.7 & 15.5 & 16 & 15.5 & 17.1 \\
\hline $\begin{array}{c}\text { I-P Mom. } \\
\text { (KNm) }\end{array}$ & 940 & 860 & 900 & 920 & 1030 \\
\hline $\begin{array}{c}\text { O-P Mom. } \\
\text { (KNm) }\end{array}$ & 675 & 720 & 660 & 740 & 820 \\
\hline
\end{tabular}

The proposed Plastic Work Curvature or PWC criterion is based on the relationship between the curvature of a characteristic load-plastic work curve and specific events in the physical evolution of a plastic failure mechanism. The results of the example analyses presented are summarized in Table 1. The limit analysis results show that the approach gives results consistent with alternative criteria for an elastic perfectly plastic material model. When applied to strain hardening analysis, the PWC criterion gives appreciably higher values of plastic load than the other criteria considered. It is suggested that this is because the PWC criterion more fully represents the effect of strain hardening on the spread of plasticity. Strain hardening inhibits plastic deformation and a strain hardening vessel requires greater load to produce the same degree of plastic deformation than would be required in a similar perfectly plastic vessel. A strain hardening vessel is therefore expected to have a higher load associated with gross plastic deformation, the plastic load, than a perfectly plastic vessel. The TES, TI and PW criteria do not represent this behavior in a consistent manner. In the nozzle example, the strain hardening plastic loads calculated by these criteria may be similar to, less than or greater than the limit load, depending on the type of load. The PWC plastic load is consistently higher than the limit load.

The nozzle under combined load problem illustrates the practical nature of the proposed criterion when the vessel is subject to multiple loads. There is no need to define a single characterizing load or deformation in order to apply the criterion. In this case, the plastic pressure is easy to identify and if used in a pressure vessel DBA would lead to an enhanced design load compared to alternative criteria. However, before the criterion can be proposed for routine design application, it is essential to establish its suitability by investigating its performance when applied to many different types of pressure vessel configurations. 


\section{NOMENCLATURE}

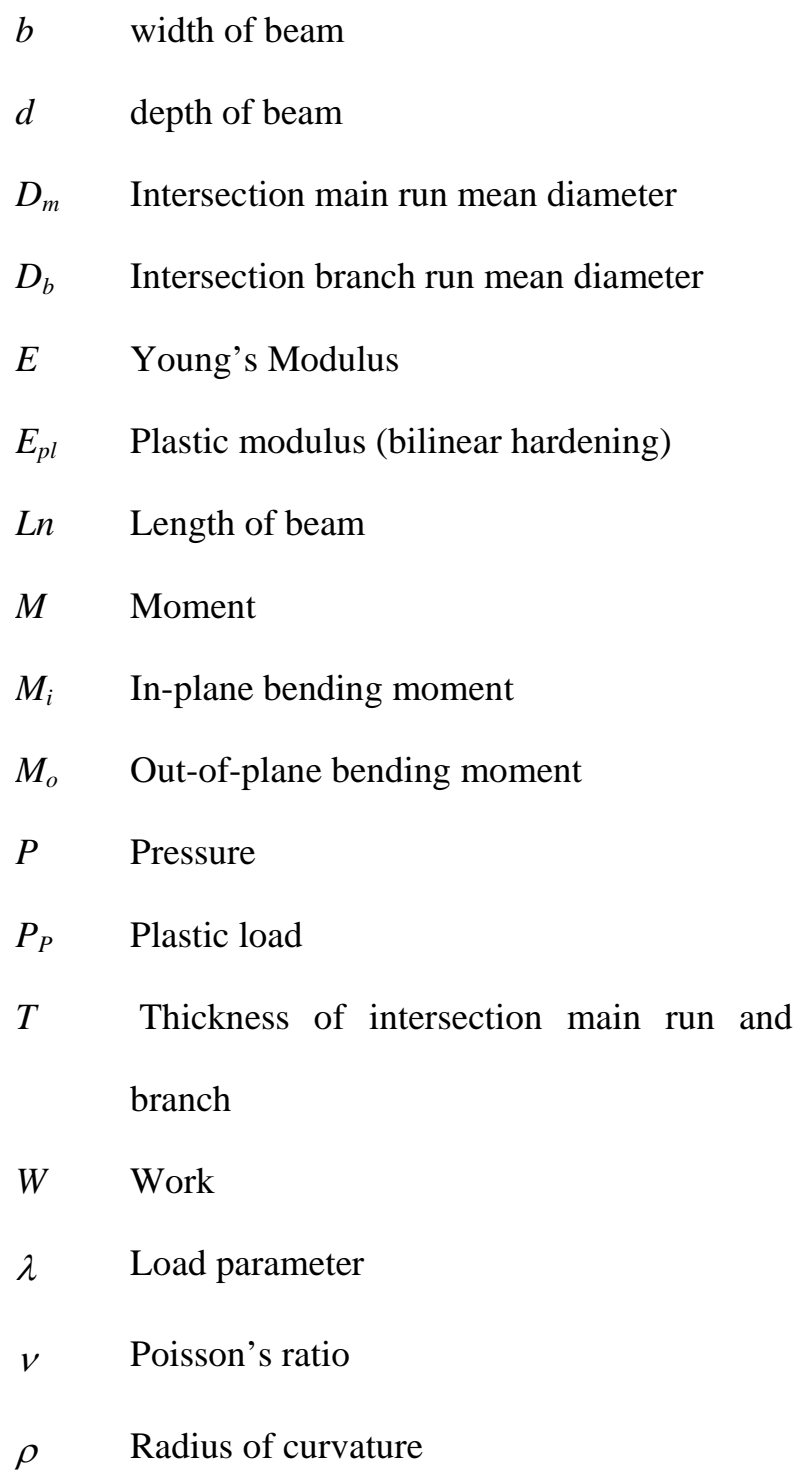

\section{Subscripts \& acronyms}
A Allowable
E $\quad$ Elastic
L $\quad$ Limit (load)
P Plastic
PW Plastic Work criterion
PWC Plastic Work Curvature criterion
TES Twice Elastic Slope criterion 
Y Yield

\section{REFERENCES}

[1] ASME, 2004, Boiler and Pressure Vessel Code Section VIII, Division 2, American Society for Mechanical Engineers, New York, USA.

[2] Gerdeen J.C., 1979, “A Critical Evaluation of Plastic Behaviour Data and a United Definition of Plastic Loads for Pressure Components,” Welding Research Bulletin No.254.

[3] Robertson A., Li H. and Mackenzie D., 2005, "Plastic collapse of pipe bends under combined internal pressure and in-plane bending,” Int. J. Pres. Ves. \& Piping, 80, pp. 407-416.

[4] Moffat D.G., Hsieh M.F. and Lynch M. , 2001, “An Assessment of ASME III and CEN TC54 Methods of Determining Plastic and Limit Loads for Pressure System Components.” J. Strain Anal, 36(3), pp. 301- 12.

[5] Save M., 1972, "Experimental verification of plastic limit analysis of torispherical and toriconical heads, Pressure vessels and piping: Design and Analysis, Vol.1 ASME pp. 382-416.

[6] Muscat M., Mackenzie D. and Hamilton R., 2003, “A work Criterion for Plastic Collapse”, Int. J. of Pressure Vessel and Piping, 80, pp. 49-58.

[7] ANSYS Version 8.0, 2003, ANSYS Inc., Canonsberg, PA 15317.

[8] Pro/Engineer, 2001, PTC, Needham, MA, USA,. 


\section{LIST OF FIGURES}

Figure 1. Twice Elastic Slope, TES, criterion.

Figure 2. Tangent Intersection, TI, criterion.

Figure 3. Plastic Work, PW, criterion.

Figure 4. Cantilever beam example structure.

Fig 5. Beam Moment-Plastic Work curve.

Figure 6. Characteristic plot of load against plastic work.

Figure 7. Moment-plastic work plot for perfectly plastic beam with curvature superimposed.

Figure 8. Finite element model of piping branch junction.

Figure 9. Multilinear hardening material model.

Figure 10. Limit analysis pressure-plastic work curve with curvature superimposed.

Figure 11. Plastic analysis pressure-plastic work plot with curvature superimposed.

Figure 12. Plastic analysis in-plane bending- plastic curve with curvature superimposed.

Figure 13. Plastic analysis out-of-plane bending -plastic work plot with curvature superimposed.

Figure 14. Plastic analysis combined loading load parameter-plastic work curve with curvature superimposed.

\section{LIST OF TABLES}

Table 1. Limit and plastic loads for the nozzle intersection under pressure, in-plane moment and outof-plane moment. 